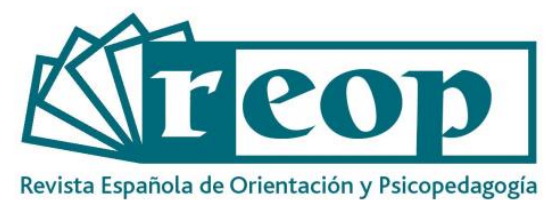

\title{
LA ASOCIACIÓN ENTRE LOS CONSTRUCTOS VOCACIONALES Y LOS TIPOS DE PERSONALIDAD DE HOLLAND EN ESTUDIANTES DE BACHILLERATO ${ }^{1}$
}

\section{THE ASSOCIATION BETWEEN VOCATIONAL CONSTRUCTS AND HOLLAND PERSONALITY TYPES IN HIGH SCHOOL STUDENTS}

\author{
María Teresa Fernández-Nistal² \\ Jairo Keven Mora-Soto \\ Santa Magdalena Mercado-Ibarra \\ Instituto Tecnológico de Sonora, Departamento de Psicología. Ciudad Obregón, México
}

\section{RESUMEN}

Desde enfoques teóricos diferentes, se ha señalado que la Rejilla Vocacional (RV) y el test Búsqueda Autodirigida (SDS) son instrumentos valiosos en el proceso de exploración vocacional. El objetivo de la investigación fue analizar la asociación entre las temáticas de los constructos vocacionales y los tipos de personalidad de Holland de estudiantes de bachillerato. Se aplicó el SDS y una versión idiográfica de la RV. Para analizar el contenido temático de los constructos vocacionales se utilizó un sistema de clasificación formado por 17 categorías agrupadas en cinco áreas temáticas (Intelectual,

\footnotetext{
1 Fuente de financiación: Esta investigación ha sido financiada por el Programa de Fomento y Apoyo a Proyectos de Investigación del Instituto Tecnológico de Sonora.

${ }^{2}$ Correspondencia: Instituto Tecnológico de Sonora, Departamento de Psicología. Calle 5 de Febrero, 818 Sur. Col. Centro, 85000, Ciudad Obregón, Sonora, México. Teléfono: 5264441090 00. Correo electrónico: teresa.fernandez@itson.edu.mx
} 
Personal, Social, Interpersonal y Laboral). Los resultados mostraron asociaciones significativas entre los tipos de personalidad y la categoría de constructos Desarrollo intelectual; los estudiantes con el tipo Investigador presentaron una frecuencia más alta en esta categoría en comparación con los de otros tipos. En la muestra de mujeres, se encontraron asociaciones significativas en las categorías Creatividad, Contribución social y Orden social: el tipo Artístico presentó con más frecuencia constructos de Creatividad, el tipo Social de Contribución social y el tipo Investigador de Orden social. En la muestra de los hombres, se obtuvieron asociaciones significativas en los constructos del área Personal, el tipo Convencional presentó una frecuencia más elevada de estos constructos. Se concluye que la RV y el SDS proporcionan información complementaria sobre los intereses y valores vocacionales del alumnado, y que existen algunos aspectos convergentes que presentan diferencias de género. La RV ofrece datos desde un enfoque cualitativo e idiográfico y el SDS desde el cuantitativo y nomotético.

Palabras clave: Constructos vocacionales, RIASEC, rejilla vocacional, búsqueda autodirigida (SDS), orientación vocacional.

\section{ABSTRACT}

It has been mentioned, from different theoretical approaches, that the Vocational Grid (VG) and the Self-Directed Search (SDS) are valuable instruments in the process of vocational exploration. The aim of the study was to analyze the association between the thematic of the vocational constructs and Holland's personality types of high school students. An idiographic version of the VG and the SDS was applied. A classification system, consisting of 17 categories grouped into five thematic areas (Intellectual, Personal, Social, Interpersonal, and Labor), was used to analyze the thematic content of the vocational constructs. The results showed significant associations between the types of vocational personality and the category "Intellectual development"; the students with the Investigative type presented a higher frequency in this category compared to those of other types. In the sample of women, significant associations were found in the categories "Creativity," "Social contribution" and "Social order": The Artistic type presented most frequently constructs of "Creativity", the Social type of "Social Contribution", and the Investigative type of "Social order". In the sample of men, significant associations were obtained in the constructs of the "Personal area", the Conventional type presented a higher frequency of these constructs. It was concluded that the VG and the SDS provide complementary information on the interests and vocational values of the students and that there are some convergent aspects that present gender differences. The VG offers data from a qualitative and idiographic approach and the SDS from the quantitative and nomothetic approach.

Key Words: Vocational constructs, RIASEC, vocational grid, the self-directed search, vocational guidance.

\section{Introducción}

En la orientación vocacional, las actividades que potencian en el alumnado la exploración de los propios intereses y valores son fundamentales para la toma de decisiones maduras que conduzcan a una inserción socio-laboral satisfactoria (Alonso-Tapia, 2012; Rivas, 1998). Desde enfoques teóricos 
diferentes, se ha señalado que la Rejilla Vocacional (RV) (Neimeyer, 1992a) y el test Búsqueda Autodirigida (SDS) (Holland, Fritzsche, y Powell, 2005) proporcionan experiencias valiosas en este proceso de autoexploración (Alfonso-Tapia, 2012; Sánchez y Valdés, 2007). En el enfoque constructivista, Savickas (1997) propuso una integración de la RV con métodos de otros modelos, como los instrumentos que evalúan los tipos de personalidad vocacional de Holland (1997), para obtener información complementaria en el proceso de asesoramiento vocacional. La RV aporta información desde una perspectiva cualitativa e idiográfica, mientras que el SDS desde la cuantitativa y nomotética, lo cual permite una compresión más rica y profunda de las preferencias y valores vocacionales del alumnado.

La RV es un instrumento de evaluación de las dimensiones y estructura de significado personal sobre el mundo académico y profesional, que permite explorar y clarificar las preferencias y valores vocacionales (Neimeyer, 1989b; Neimeyer, 1992a; Savickas, 1997). Este instrumento se encuadra en la teoría de los constructos personales (Kelly, 1955/1991), cuyo postulado fundamental es que "los procesos de una persona se canalizan psicológicamente por las formas en que anticipa los acontecimientos" (p. 32). Según Kelly la anticipación de los acontecimientos se produce mediante la construcción de dimensiones de interpretación de la experiencia, denominadas constructos personales (Botella y Feixas, 2008; Neimeyer, 1989b). Los constructos personales son de naturaleza bipolar; permiten asimilar dos eventos y, a la vez, contrastarlos con un tercero que representa el otro polo del constructo; son finitos y se interconectan entre ellos en sistemas jerárquicos; son idiográficos y tienen como misión fundamental la anticipación significativa y predicción de la experiencia (Botella y Feixas, 2008; Feixas y Cornejo, 1996; Neimeyer, 1989a).

Kelly consideró la experiencia vocacional como uno de los aspectos fundamentales de la vida de una persona: "La vocación es uno de los principales medios por los que se define y da significado a nuestro rol en la vida" (Kelly, 1955/1991, p. 751) y postuló la existencia de un "sistema de constructos vocacionales" (Kelly, 1955/1991, p. 741), que consiste en una matriz idiográfica e interrelacionada de constructos bipolares cuyo foco de conveniencia son los eventos profesionales y vocacionales (Neimeyer, 1989a). Este sistema comprende los constructos que son utilizados por el individuo para interpretar y sistematizar la experiencia vocacional, y planificar los comportamientos relacionados con el ámbito vocacional y profesional (Neimeyer, 1989a; Savickas, 1997).

El origen de la RV se sitúa en el test de Repertorios de Constructos de Roles (REP), un instrumento clínico ideado por Kelly (1955/1991) para obtener constructos personales sobre el mundo interpersonal. El procedimiento de administración del REP constaba de tres pasos: elección de los elementos, obtención de los constructos y representación de los datos, que podía ser en forma de lista o de rejilla. Desde entonces, esta técnica ha sido muy utilizada en diversos ámbitos de la psicología y otras disciplinas (Saul et al., 2012; Taylor, 2016), y se han generado distintas versiones que varían en el procedimiento de administración y análisis (Botella y Feixas, 2008).

La mayoría de las investigaciones realizadas en el área vocacional han utilizado la versión estandarizada de la RV (Neimeyer, 1992b; Paszkowska-Rogacz y Kabzińska, 2012; Rivas, Rocabert, y López, 1998). En esta versión se proporcionan, de antemano, los elementos y/o constructos en lugar de solicitar a las personas que ellas mismas los elaboren. Sin embargo, este procedimiento se ha criticado por su incongruencia con el fundamento fenomenológico y constructivista de la teoría de los constructos personales (Feixas y Cornejo, 1996).

El análisis de la RV se puede realizar desde un enfoque cualitativo (de contenido) y cuantitativo (de la estructura). El primero consiste en un análisis clínico de los datos (Kelly, 1955/1991); algunos aspectos que se pueden estudiar desde el enfoque cualitativo son el número y contenido temático de los constructos y elementos, y el análisis formal de los constructos. El segundo enfoque, el análisis de la estructura, hace referencia al estudio de las relaciones cuantitativas entre constructos y entre 
elementos a través de pruebas estadísticas, del que se han derivado diversos índices de medida cognitivos (Botella y Feixas, 2008).

La mayoría de las investigaciones realizadas en el área vocacional se han enfocado en el análisis de la estructura cognitiva (Martínez, 2005; Neimeyer, 1992b). Los índices más estudiados han sido la diferenciación e integración (Mau, 1997; Vilhjálmsdóttir, 2010). Existen pocas investigaciones realizadas con un abordaje cualitativo de los datos y, concretamente, que hayan elaborado sistemas de categorías para la evaluación del contenido temático de los constructos vocacionales (Neimeyer, 1989b), los cuales pueden ser una herramienta muy útil en el análisis de las versiones idiográficas de la RV. Únicamente, se han encontrado la clasificación de Rivas y colaboradores (Rivas y Ardit, 1985, citado en Martínez, 2005), obtenida a partir de una muestra de preuniversitarios españoles y cuyo objetivo principal fue diseñar una versión estandarizada de la RV, y otra, más reciente, realizada por Fernández, Tuset y Cuervo (2017), obtenida de una muestra de alumnado indígena de México.

Por otro lado, el SDS (Holland et al., 2005) es un inventario de autoexploración sobre los intereses y aspiraciones vocacionales. Su aplicación representa una experiencia de aprendizaje, en la cual la persona se inicia en un proceso de exploración y de toma de decisiones relacionadas con el desarrollo de la carrera (Martínez, 2007). Este instrumento está formado por preguntas acerca de aspiraciones ocupacionales, actividades y ocupaciones preferidas, y sobre la autovaloración de capacidades y habilidades. Es uno de los instrumentos de orientación de carrera más utilizados en muchos países (Martínez, 2007; Nauta, 2010). En México, existe una adaptación y estandarización del SDS (Holland et al., 2005) y su uso es muy frecuente en los servicios de asesoría vocacional (Sanchez y Valdés, 2007).

EI SDS está basado en la teoría de los tipos de personalidad vocacional y ambientes de trabajo de Holland (1997), cuyo principio fundamental es que los intereses vocacionales son expresión de la personalidad de los individuos. De acuerdo con esta teoría, las personas pueden ser categorizadas en uno o una combinación de los siguientes tipos de personalidad vocacional: realista $(R)$, investigador (I), artístico (A), social (S), emprendedor (E) y convencional (C); los cuales se representan en este orden en un modelo hexagonal que indica las relaciones entre estos tipos. Asimismo, para este autor la personalidad y el ambiente de trabajo interaccionan entre sí influyendo de manera bidireccional y los ambientes de trabajo se pueden describir, por sus semejanzas, en uno o en una combinación de los tipos RIASEC. Según Holland (1997), las personas buscan ambientes de trabajo que les permitan ejercer sus habilidades y capacidades, expresar sus actitudes y valores, y asumir problemas y roles de su agrado. EI SDS proporciona información sobre el código personal (de acuerdo al orden de las puntuaciones obtenidas en los tipos de personalidad) y el de aspiraciones ocupacionales, además de los índices de diferenciación, congruencia y consistencia.

Cada uno de los tipos de personalidad vocacional representa una compleja integración de aptitudes, opiniones acerca de uno mismo, rasgos de personalidad, intereses, valores, metas, identificaciones y perspectivas acerca del mundo (Holland et al., 2005). La conceptualización de que Ios tipos RIASEC son expresiones amplias de la personalidad, tuvo como consecuencia el desarrollo de una línea de investigación dirigida a estudiar las relaciones de esta tipología con otras variables psicológicas. Los primeros estudios analizaron el vínculo de los tipos de personalidad vocacional con los valores y metas, investigaciones posteriores relacionaron la tipología con las dimensiones de la personalidad Big Five, auto-eficacia y las demandas ambientales (Nauta, 2010).

Son escasas las investigaciones que han analizado la relación entre variables de la teoría de Holland con las de la teoría de los constructos personales de Kelly aplicada al área vocacional. Las investigaciones que existen sobre este tema se han enfocado en estudiar la complejidad cognitiva a través de la RV en personas con diferentes tipos de personalidad vocacional (Bodden, 1970; Winer, Warren, Dailey, y Hiesberger, 1980), en validar ciertos índices cognitivos de la RV (conflicto, discriminación e intensidad) a partir de las puntuaciones en los índices de diferenciación y 
consistencia de Holland (Hay, 1985) y en obtener evidencia de validez de los tipos de personalidad RIASEC a partir de la RV (Paszkowska-Rogacz y Kabzińska, 2012). La cuestión de la relación entre los tipos de personalidad de Holland y la temática de los constructos vocacionales no ha sido abordada en estas investigaciones.

Teniendo en cuenta la relevancia y utilidad de la RV y el SDS en el proceso de exploración vocacional y que la aplicación conjunta de ambos instrumentos puede aportar información desde distintos puntos de vista para enriquecer la comprensión del alumnado sobre sus propios intereses, motivaciones y valores, se planteó el objetivo de analizar la asociación entre las temáticas de los constructos vocacionales obtenidos en la RV y los tipos de personalidad vocacional de Holland de estudiantes de bachillerato.

Al margen de las diferencias entre los constructos personales y los tipos de personalidad, y de las teorías que los fundamentan; partimos de la conjetura de que se encontrarán aspectos convergentes en la medida en que ambos son concepciones de naturaleza global. Por un lado, los tipos de personalidad de Holland están integrados por opiniones acerca de uno mismo, rasgos de personalidad, intereses, valores, metas, identificaciones y perspectivas acerca del mundo (Holland et al., 2005) y, por otro lado, el sistema de constructos personales consiste en dimensiones de interpretación personal sobre un determinado ámbito de la experiencia, que se construyen a través de un proceso global en el que intervienen distintos elementos, como los valores (o constructos nucleares), autoconceptos o hipótesis sobre uno mismo, preocupaciones e intereses relevantes. (Botella y Feixas, 2008; Savickas, 1997).

\section{Método}

\section{Muestra}

La muestra está formada por 169 estudiantes (79 hombres y 90 mujeres) de dos centros de educación públicos situados en Ciudad Obregón (México), que cursaban de $2^{\circ}$ a $6^{\circ}$ semestre de bachillerato. La edad de los participantes oscila entre los 15 y 19 años $(M=16.3, S D=.91)$ y el nivel socioeconómico de sus familias es medio-bajo. El muestreo fue intencional por criterio (Izcara, 2007); se contactó de manera deliberada con la dirección de dos centros de bachillerato, los cuales aceptaron participar en la investigación y se solicitó al personal de orientación educativa que seleccionara al alumnado interesado en colaborar y con capacidad y motivación en la asignatura de orientación educativa, con tal de asegurar su participación en la investigación y la riqueza de la información proporcionada.

\section{Instrumentos}

Se aplicaron la versión de la RV de Padilla (2001) y la adaptación para México del SDS forma R (Holland et al., 2005). En esta versión de la rejilla el alumnado proporciona por sí mismo los elementos y los constructos vocacionales. La administración de la RV consta de tres fases. La 
primera consiste en la elaboración de elementos, donde el estudiante tiene que mencionar entre 8 y 10 actividades profesionales que le gustaría desempeñar en un futuro, independientemente de la posibilidad real de ejercerlas, y una profesión que no le gustaría. La segunda fase consiste en la obtención de constructos, en la cual se presentan al estudiante parejas de los elementos y se le pregunta en qué se parecen y en qué se diferencian estas actividades profesionales. La tercera fase corresponde a la asignación de puntuaciones, donde el estudiante valora los elementos en relación con cada polo del constructo a partir de una escala de 5 puntos.

Por su parte, el SDS es un inventario de personalidad e intereses vocacionales autoaplicable y autointerpretable. Está dividido en cuatro secciones (escalas SDS, cómo organizar tus respuestas, aspiraciones ocupacionales y lo que significa tu código personal). Las escalas SDS están formadas por 228 ítems divididos en cuatro apartados: actividades, habilidades, ocupaciones y calificación de capacidades. En relación con sus propiedades psicométricas, en la versión estadounidense se obtuvieron de las puntuaciones totales coeficientes de fiabilidad test-retest altos (entre .76 y .89) e índices de consistencia interna excelentes (de .90 a .94). En cuanto a la validez concurrente y predictiva, se halló un nivel general de aciertos elevado (54.7\%) entre las puntuaciones más altas del código personal y el de aspiración.

\section{Procedimiento}

Un psicólogo aplicó los instrumentos de forma colectiva a grupos de 10 estudiantes. Se llevaron a cabo dos sesiones de administración con estos grupos; en la primera se mantuvo una conversación con el alumnado acerca de sus expectativas profesionales y educativas con el objetivo de establecer rapport y se aplicó el SDS, en la segunda se aplicó la RV. Las sesiones se llevaron a cabo en el horario lectivo, tuvieron una duración aproximada de 50 minutos y se realizaron en el ciclo escolar 2016-2017. El estudio cumplió con las normas del código de ética de la American Psychological Association (2017): autorización institucional, consentimiento informado del alumnado, confidencialidad y devolución de los resultados. Se prescindió del consentimiento informado de la familia con base en las normas 8.05 y 9.03 de este código, puesto que la aplicación de los instrumentos se integró dentro de las actividades educativas de la asignatura orientación educativa.

\section{Procedimiento en el análisis de los datos}

Para analizar el contenido temático de los constructos vocacionales se utilizó el sistema de clasificación de Fernández et al. (2017), el cual está formado por 17 categorías de constructos significativos agrupados en cinco áreas temáticas (Intelectual, Personal, Social, Interpersonal y Laboral) y tres categorías de constructos no significativos (situacionales, superficiales y vagos). Los constructos significativos proporcionan información sobre los intereses, motivaciones y valores personales y vocacionales, mientras que los no significativos contienen información concreta e irrelevante. Este sistema de clasificación se construyó a partir de las RV de una muestra de alumnado de secundaria indígena de México.

Dadas las diferencias culturales de esa muestra con la del presente estudio, en el proceso de análisis se adoptó una postura abierta a la inclusión de nuevas categorías en el sistema de clasificación. En primer lugar, dos investigadores codificaron independientemente los constructos de las RV a partir de este sistema de clasificación. La concordancia interevaluadores fue alta (coeficiente Kappa=.83). En los casos donde hubo discrepancias, se alcanzó un consenso a partir de revisiones conjuntas. En segundo lugar, se realizó un análisis cualitativo de contenido de los constructos incluidos en las categorías "otros" de cada una de las cinco áreas temáticas del sistema de 
clasificación, para identificar nuevas categorías de constructos significativos con una frecuencia suficiente. Este análisis permitió identificar dos nuevas categorías: "Defensa del estado" incluida en el área Social e "Independencia en el trabajo" en el área Laboral. Estas dos categorías se definieron e integraron en el sistema de clasificación. EI SDS se corrigió según los criterios del manual de esta prueba.

Las puntuaciones de ambos instrumentos se ingresaron en el programa IBM SPSS Statistics 21 y se sometieron a un procedimiento de doble comprobación. Se aplicó la prueba Chi-cuadrado para analizar la asociación entre la frecuencia de constructos vocacionales y los tipos de personalidad vocacional, y para explorar diferencias según el sexo y semestre del alumnado.

\section{Resultados}

\section{Los tipos de personalidad vocacional}

En la Tabla 1 se presenta la frecuencia de estudiantes clasificados en los tipos de personalidad vocacional según la puntuación más alta obtenida en el SDS. Once estudiantes obtuvieron la misma puntuación en más de un tipo de personalidad y no se consideraron en los resultados. El tipo de personalidad $S$ presentó la frecuencia más alta de estudiantes, en segundo lugar el tipo $E$, en tercer lugar el I y en cuarto el A. Los tipos de personalidad con menor frecuencia fueron el $\mathrm{R}$ y el $\mathrm{C}$.

\section{Tabla 1}

Frecuencias de estudiantes en los tipos de personalidad vocacional según el semestre y el sexo

\begin{tabular}{llllllllc}
\hline Tipos de personalidad & \multicolumn{4}{c}{ Semestres } & \multicolumn{2}{c}{ Sexo } \\
vocacional & $\mathbf{2}$ & $\mathbf{3}^{\mathbf{0}}$ & $\mathbf{4}$ & $\mathbf{5}$ & $\mathbf{6}$ & Mujeres & Hombres & Total \\
\hline Realista (R) & 4 & 2 & 2 & & 4 & & $12(16 \%)$ & $12(8 \%)$ \\
Investigador (I) & 6 & 3 & 7 & 11 & & $10(12 \%)$ & $17(23 \%)$ & $27(17 \%)$ \\
Artístico (A) & 8 & 6 & 7 & & 1 & $10(12 \%)$ & $12(16 \%)$ & $22(14 \%)$ \\
Social (S) & 21 & 10 & 14 & 5 & 7 & $44(52 \%)$ & $13(18 \%)$ & $57(36 \%)$ \\
Emprendedor (E) & 11 & 4 & 5 & 8 & 6 & $17(20 \%)$ & $17(23 \%)$ & $34(21 \%)$ \\
Convencional (C) & 3 & & & 3 & & $3(4 \%)$ & $3(4 \%)$ & $6(4 \%)$ \\
Total & 53 & 25 & 35 & 27 & 18 & 84 & 74 & 158 \\
\hline
\end{tabular}

Fuente: elaboración propia.

Se hallaron diferencias significativas en los tipos de personalidad según el sexo, $X^{2}(5, N=158)=$ 30.34, $p<.005$, con un tamaño del efecto (TE) mediano ( $V$ de Cramer=.44). Los resultados de las pruebas Chi-cuadrado para cada tipo de personalidad mostraron diferencias significativas según el sexo únicamente en el tipo $S, X^{2}(1, N=158)=20.68, p<.005$, con un TE mediano ( $V$ de Cramer=.36), y en el tipo $\mathrm{R}, \mathrm{X}^{2}(1, N=158)=14.74, p<.005$, con un TE mediano ( $V$ de Cramer=.31); un mayor porcentaje de mujeres se clasificaron en el tipo de personalidad $S$ que los hombres, mientras que estos se clasificaron con más frecuencia en el tipo $R$ que las mujeres. Por otro lado, no se encontraron diferencias significativas según los semestres, $X^{2}(5, N=158)=5.52, p>.1$

REOP. Vol. 31, no1, 1er Cuatrimestre, 2020, pp. 10 - 25 [ISSN electrónico: 1989-7448] 


\section{Contenido temático de los constructos vocacionales}

Se obtuvieron un total de 1499 constructos, de los cuales $929(62 \%)$ fueron significativos, el resto $(38 \%)$ se clasificaron como no significativos (situacionales, superficiales y vagos). Estos últimos no se han considerado en esta investigación con base en la recomendación de Kelly (1955/1991) de evitar en el análisis de las rejillas los constructos no significativos.

\section{Tabla 2}

Frecuencia y porcentajes (entre paréntesis) de constructos vocacionales en el sistema de clasificación según los tipos de personalidad de la muestra

\begin{tabular}{|c|c|c|c|c|c|c|c|c|}
\hline \multirow{2}{*}{\multicolumn{2}{|c|}{$\begin{array}{l}\text { Sistema de clasificación de los } \\
\text { constructos vocacionales }\end{array}$}} & \multicolumn{6}{|c|}{ Tipos de personalidad vocacional } & \multirow{3}{*}{$\begin{array}{c}\text { Total } \\
\boldsymbol{N}=158 \\
227(24) \\
97(10)\end{array}$} \\
\hline & & \multirow{3}{*}{$\begin{array}{c}\begin{array}{c}\mathbf{R} \\
\boldsymbol{n = 1 2}\end{array} \\
13(22) \\
13(22)\end{array}$} & \multirow{2}{*}{$\begin{array}{c}\mathbf{I} \\
\boldsymbol{n}=\mathbf{2 7} \\
76(42) \\
16(9)\end{array}$} & \multirow{2}{*}{$\begin{array}{c}\mathbf{A} \\
\boldsymbol{n = 2 2} \\
19(14) \\
22(16)\end{array}$} & \multirow{2}{*}{$\begin{array}{c}\mathbf{S} \\
\boldsymbol{n}=57\end{array}$} & \multirow{2}{*}{$\begin{array}{c}\begin{array}{c}\mathbf{E} \\
\boldsymbol{n}=\mathbf{3 4}\end{array} \\
50(27) \\
19(10)\end{array}$} & \multirow{2}{*}{$\begin{array}{c}\mathbf{C} \\
\boldsymbol{n}=6 \\
4(14) \\
2(7)\end{array}$} & \\
\hline \multirow{3}{*}{. } & -Desarrollo intelectual & & & & & & & \\
\hline & -Otros & & & $3(2)$ & & & & $3(.3)$ \\
\hline & Total & $26(44)$ & $92(51)$ & $44(32)$ & $90(26)$ & $69(38)$ & $6(21)$ & $327(35)$ \\
\hline \multirow{6}{*}{ 离 } & -Autoafirmación y/o realización & & $1(.5)$ & & $5(1.5)$ & $2(1)$ & $3(11)$ & $11(1)$ \\
\hline & -Responsabilidad & $1(2)$ & $8(4)$ & $5(4)$ & $15(4)$ & $9(5)$ & $2(7)$ & $40(4)$ \\
\hline & -Prestigio & & & $1(1)$ & & $2(1)$ & & $3(.3)$ \\
\hline & -Popularidad & & & $2(1)$ & $2(.6)$ & $3(2)$ & & $7(.7)$ \\
\hline & -Glamour & & & & $5(1.5)$ & $5(3)$ & & $10(1)$ \\
\hline & Total & $1(2)$ & $9(5)$ & $8(6)$ & $27(8)$ & $22(12)$ & $5(18)$ & $72(8)$ \\
\hline \multirow{5}{*}{ 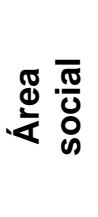 } & -Contribución social & $19(32)$ & $51(28)$ & $53(39)$ & $162(47)$ & $67(37)$ & $13(46)$ & 365 (39) \\
\hline & -Orden social & $8(13)$ & $16(9)$ & $13(10)$ & $24(7)$ & $6(3)$ & $1(4)$ & $68(7)$ \\
\hline & -Defensa del estado ${ }^{a}$ & $1(2)$ & $1(.5)$ & $2(1)$ & $8(2.3)$ & & $1(4)$ & $13(1)$ \\
\hline & -Otros & & & $1(1)$ & & & & $1(.1)$ \\
\hline & Total & $28(47)$ & $68(38)$ & $69(51)$ & $194(56)$ & $73(40)$ & $15(54)$ & $447(48)$ \\
\hline \multirow{5}{*}{ 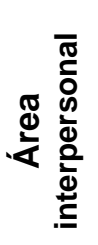 } & -Relaciones interpersonales & $2(3)$ & $5(3)$ & $5(4)$ & $19(6)$ & $8(4)$ & $2(7)$ & $41(4)$ \\
\hline & -Autoridad & $1(2)$ & & $1(1)$ & $2(.6)$ & $4(2)$ & & $8(.8)$ \\
\hline & -Obediencia & & & & $1(.3)$ & & & $1(.1)$ \\
\hline & -Otros & & & & $1(.3)$ & & & $1(.1)$ \\
\hline & Total & $3(5)$ & $5(3)$ & $6(4)$ & $23(7)$ & $12(6)$ & $2(7)$ & $51(5)$ \\
\hline \multirow{7}{*}{ 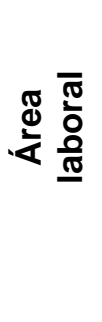 } & -Retribución económica & $1(2)$ & $1(.5)$ & $2(1)$ & $1(.3)$ & $5(3)$ & & $10(1)$ \\
\hline & -Seguridad laboral & & $2(1)$ & $3(2)$ & $2(.6)$ & & & $7(.7)$ \\
\hline & -Sacrifico en el trabajo & & & $3(2)$ & $2(.6)$ & & & $5(.5)$ \\
\hline & -Independencia en el trabajo ${ }^{\mathrm{b}}$ & & & $1(1)$ & $4(1)$ & $2(1)$ & & $7(.7)$ \\
\hline & -Otros & & $3(2)$ & & & & & $3(.3)$ \\
\hline & Total & $1(2)$ & $6(3)$ & $9(7)$ & $9(3)$ & $7(4)$ & & $32(3)$ \\
\hline & TOTAL & 59 & 180 & 136 & 343 & 183 & 28 & 929 \\
\hline
\end{tabular}

Notas. a: Incluye los constructos que mencionan actividades dirigidas a salvaguardar la soberanía del país ("lucha por el país, su patria vs. no lucha", "ayudar a salvar tu nación vs. no te preocupas por tu nación"). •: Incluye los constructos que hacen referencia a la situación de ser autónomo en el trabajo ("tendría un puesto de trabajo propio vs. tendría un trabajo en el gobierno para hacer construcciones públicas", "en ambos tienen su propio despacho vs. trabajan en lugares de otras personas"). Fuente: elaboración propia. 
En la Tabla 2 se presenta la frecuencia de constructos en el sistema de clasificación obtenida por la muestra según su tipo de personalidad vocacional. Se puede observar que las categorías de constructos vocacionales más frecuentes fueron, en primer lugar, Contribución social (39\%), en segundo lugar Desarrollo intelectual (24\%), en tercer lugar Creatividad (10\%) y en cuarto lugar Orden social (7\%). El resto de las categorías obtuvieron porcentajes iguales o inferiores al $4 \%$. Por áreas, la más frecuente fue la Social (48\%) y en segundo lugar la Intelectual (35\%). El resto de las áreas alcanzó porcentajes bajos, el área Personal 8\%, la Interpersonal 5\% y la Laboral 3\%.

Se hallaron diferencias significativas en las frecuencias de constructos de las áreas temáticas según el sexo, $X^{2}(4, N=929)=16.64, p<.005$, con un TE pequeño ( $V$ de Cramer=.13). Los resultados de la prueba Chi-cuadrado para cada área mostraron diferencias significativas según esta variable únicamente en tres áreas: la Intelectual, $X^{2}(1, N=929)=6.99, p<.01$, con un TE muy pequeño ( $V$ de Cramer=.09); la Social, $X^{2}(1, N=929)=12.26, p<.005$, con un TE pequeño ( $V$ de Cramer=.11); y la Laboral, $X^{2}(1, N=929)=6.47, p<.025$, con un TE muy pequeño ( $V$ de Cramer=.08). Los hombres presentaron un porcentaje más alto de constructos en el área Intelectual (40\%) que las mujeres (31\%), mientras que estas obtuvieron un porcentaje más elevado de constructos en el área Social $(53 \%)$ que los hombres (42\%), y en el área Laboral los hombres presentaron más constructos $(5 \%)$ que las mujeres $(2 \%)$. No se encontraron diferencias significativas según el semestre, $X^{2}(4, N=929)=$ 3.33, $p>.01$.

\section{Asociación entre los tipos de personalidad y las temáticas de los constructos vocacionales}

En la Tabla 3 se presenta la intersección entre los tipos de personalidad vocacional y el sistema de clasificación de los constructos vocacionales según el sexo. La categoría Contribución social fue la más frecuente en los tipos de personalidad $\mathrm{R}, \mathrm{A}, \mathrm{S}, \mathrm{E}$ y $\mathrm{C}$ de ambos sexos, con porcentajes que van en las mujeres del $35 \%$ en el tipo $\mathrm{C}$ al $51.5 \%$ en el $\mathrm{S}$ y en los hombres del $32 \%$ en el tipo R al $64 \%$ en el tipo C. En cambio, la categoría más frecuente en el tipo de personalidad I fue Desarrollo Intelectual tanto en las mujeres (37\%) como en los hombres (45\%).

La categoría Desarrollo intelectual fue la segunda más frecuente en los hombres y mujeres con los tipos de personalidad S (18\% y $19 \%$, respectivamente) y $E$ (27\% en ambos sexos), en los hombres con el tipo A (15\%) y en las mujeres con el tipo C (23\%). En las mujeres con el tipo de personalidad A la segunda categoría más frecuente fue Creatividad (22\%) y en los hombres con el tipo C Responsabilidad (18\%). En los hombres con el tipo $\mathrm{R}$ la segunda categoría más frecuente estuvo compartida por Desarrollo intelectual y Creatividad (ambas con el 22\%). Debido a las diferencias de género halladas en los tipos de personalidad y en el sistema de clasificación de los constructos vocacionales, se llevaron a cabo análisis independientes para cada sexo en el estudio de la asociación entre la distribución de frecuencias de los constructos vocacionales y los tipos de personalidad de Holland (ver tabla 3). Los resultados de esta asociación permitieron rechazar la hipótesis de independencia en la categoría Desarrollo intelectual en ambos sexos y en las categorías Creatividad, Contribución social y Orden social únicamente en la muestra de mujeres. Por áreas, se rechazó la prueba de independencia en la Intelectual y Social en ambos sexos, y en la Personal solo en los hombres.

En la categoría Desarrollo intelectual se encontraron diferencias significativas en la distribución de frecuencias según los tipos de personalidad en la muestra de los hombres, con un TE pequeño ( $V$ de Cramer=.28), y en la de mujeres, con un TE pequeño ( $V$ de Cramer=.17). Las mujeres y hombres con el tipo de personalidad I presentaron una frecuencia más elevada de esta categoría de constructos (37\% y $45 \%$, respectivamente) en comparación con los de otros tipos de personalidad, 
sobre todo con los de los tipos A (15\% en los hombres y $13 \%$ en las mujeres) y $S$ (18\% en los hombres y $19 \%$ en las mujeres). Respecto a la categoría Creatividad, en la muestra de mujeres se obtuvieron diferencias significativas según los tipos de personalidad vocacional, con un TE pequeño ( $V$ de Cramer=.19); las mujeres con el tipo A presentaron un porcentaje más alto de esta categoría de constructos (22\%) que las de otros tipos de personalidad, especialmente con las de los tipos S (5\%) e I (8\%).

\section{Tabla 3}

Porcentajes de constructos y asociación entre el sistema de clasificación de los constructos vocacionales y los tipos de personalidad de la muestra de hombres $(H)$ y mujeres $(M)$

\begin{tabular}{|c|c|c|c|c|c|c|c|c|c|c|}
\hline \multirow{2}{*}{\multicolumn{2}{|c|}{$\begin{array}{l}\text { Sistema de clasificación de } \\
\text { los constructos vocacionales }\end{array}$}} & \multicolumn{6}{|c|}{ Tipos de personalidad vocacional } & \multirow{3}{*}{\multicolumn{2}{|c|}{$\begin{array}{c}\mathbf{H} \\
\begin{array}{c}\mathrm{X}^{2} \\
(g l=5)\end{array}\end{array}$}} & \multirow{3}{*}{$\begin{array}{c}\mathbf{M} \\
\begin{array}{c}\mathbf{X}^{2} \\
(g l=4)\end{array}\end{array}$} \\
\hline & & \multirow{2}{*}{$\begin{array}{c}R \\
H / M \\
\end{array}$} & \multirow{2}{*}{$\begin{array}{c} \\
H / M \\
\end{array}$} & \multirow{2}{*}{$\begin{array}{c}\mathrm{A} \\
\mathrm{H} / \mathrm{M} \\
\end{array}$} & \multirow{2}{*}{$\begin{array}{c}\mathrm{S} \\
\mathrm{H} / \mathrm{M} \\
\end{array}$} & \multirow{2}{*}{$\begin{array}{c}\mathrm{E} \\
\mathrm{H} / \mathrm{M} \\
\end{array}$} & \multirow{2}{*}{$\begin{array}{c}C \\
H / M \\
\end{array}$} & & & \\
\hline & & & & & & & & & & \\
\hline \multirow{4}{*}{ 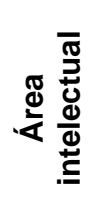 } & -Desarrollo intelectual & $22 /$ & $45 / 37$ & $15 / 13$ & $18 / 19$ & $27 / 27$ & $/ 23$ & $110 / 117$ & $31.18^{* * *}$ & $15.18^{* * *}$ \\
\hline & -Creatividad & $22 /$ & $9 / 8$ & $11 / 22$ & $14 / 5$ & $9 / 10$ & $/ 12$ & $50 / 47$ & $8.29^{b}$ & $18.28^{* * *}$ \\
\hline & -Otros & & & $3 / 1$ & & & & $2 / 1$ & a & a \\
\hline & Total & $44 /$ & $54 / 45$ & $29 / 36$ & $32 / 24$ & $36 / 37$ & $/ 35$ & $162 / 165$ & $23.11^{* * *}$ & $14.40^{* *}$ \\
\hline \multirow{5}{*}{ 这 } & $\begin{array}{l}\text {-Autoafirmación y/o } \\
\text { realización }\end{array}$ & & $1 /$ & & $4 / 1$ & 12 & $9 / 12$ & $5 / 6$ & a & a \\
\hline & -Responsabilidad & 2/ & $5 / 3$ & $3 / 4$ & $2 / 4$ & $3 / 6$ & $18 /$ & $15 / 25$ & a & a \\
\hline & -Prestigio & & & $/ 1$ & & $3 /$ & & $2 / 1$ & a & a \\
\hline & -Popularidad & & & $2 / 1$ & $4 / 1$ & 4/ & & $4 / 3$ & a & $\mathrm{a}$ \\
\hline & $\begin{array}{l}\text {-Glamour } \\
\text {-Otros }\end{array}$ & & & & $/ 1$ & $\begin{array}{c}1 / 4 \\
1 /\end{array}$ & & $\begin{array}{c}4 / 6 \\
1 /\end{array}$ & $\begin{array}{l}\mathrm{a} \\
\mathrm{a}\end{array}$ & $\begin{array}{l}\mathrm{a} \\
\mathrm{a}\end{array}$ \\
\hline \multirow{5}{*}{ 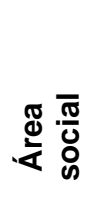 } & Total & $2 /$ & $6 / 3$ & $5 / 7$ & $10 / 7$ & $12 / 12$ & $27 / 12$ & $31 / 41$ & $12.90^{\star * *}$ & $5.43^{\mathrm{b}}$ \\
\hline & -Contribución social & $32 /$ & $30 / 25$ & $38 / 40$ & $33 / 51.5$ & $35 / 38$ & $64 / 35$ & $138 / 227$ & $5.71^{b}$ & $18.10^{* * *}$ \\
\hline & -Orden social & $13 /$ & $2 / 22$ & $9 / 10$ & $6 / 7$ & $3 / 4$ & 9/ & $24 / 44$ & a & $20.36^{* * *}$ \\
\hline & $\begin{array}{l}\text {-Defensa del estado a } \\
\text {-Otros }\end{array}$ & $2 /$ & $1 /$ & $\begin{array}{l}3 / \\
/ 1\end{array}$ & $5 / 1.5$ & & 16 & $\begin{array}{c}8 / 5 \\
/ 1\end{array}$ & $\begin{array}{l}\mathrm{a} \\
\mathrm{a}\end{array}$ & $\begin{array}{l}\mathrm{a} \\
\mathrm{a}\end{array}$ \\
\hline & Total & $47 /$ & $33 / 47$ & $50 / 51$ & $44 / 60$ & $38 / 42$ & $73 / 41$ & $170 / 277$ & $11.53^{*}$ & $13.06^{*}$ \\
\hline \multirow{5}{*}{ 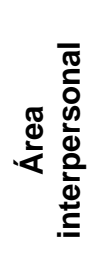 } & $\begin{array}{l}\text {-Relaciones } \\
\text { interpersonales }\end{array}$ & $3 /$ & $3 / 1.5$ & $4 / 3$ & $5 / 5.5$ & $5 / 4$ & $/ 12$ & $17 / 24$ & a & a \\
\hline & -Autoridad & $2 /$ & & $/ 1$ & $/ 1$ & $4 / 1$ & & $4 / 4$ & a & a \\
\hline & -Obediencia & & & & $/ .5$ & & & $/ 1$ & a & a \\
\hline & -Otros & & & & $1 /$ & & & $1 /$ & a & a \\
\hline & Total & $5 /$ & $3 / 1.5$ & $4 / 4$ & $7 / 7$ & $9 / 5$ & $/ 12$ & $22 / 29$ & a & a \\
\hline \multirow{7}{*}{ 悉 } & -Retribución económica & $2 /$ & $/ 1.5$ & $2 / 1$ & $1 /$ & $4 / 2$ & & $6 / 4$ & a & a \\
\hline & -Seguridad laboral & & 2/ & $4 /$ & $2 /$ & & & $7 /$ & a & a \\
\hline & -Sacrifico en el trabajo & & & $4 /$ & $1 / .5$ & & & $4 / 1$ & a & a \\
\hline & $\begin{array}{l}\text {-Independencia en el } \\
\text { trabajo }{ }^{b}\end{array}$ & & & $2 /$ & $1 / 1.5$ & 12 & & $2 / 5$ & a & a \\
\hline & -Otros & & $2 / 1.5$ & & & & & $2 / 1$ & a & a \\
\hline & Total & 2/ & $4 / 3$ & $12 / 1$ & $6.5 / 2$ & $4 / 4$ & & $21 / 11$ & a & a \\
\hline & TOTAL & $59 /$ & $116 / 64$ & $66 / 70$ & $77 / 266$ & $77 / 106$ & $11 / 17$ & $406 / 523$ & & \\
\hline
\end{tabular}

Nota: ${ }^{a}$ No se calculó la prueba Chi-cuadrado porque se incumple la condición de que las frecuencias esperadas menores que 5 no superen el $20 \%$ del total de frecuencias de la tabla. ${ }^{\mathrm{b}} p>.05 ;{ }^{*} p<.05 ;{ }^{* \star} p<.01 ;{ }^{* \star *} p<.005$. Fuente: elaboración propia. 
En la categoría Contribución social las mujeres presentaron diferencias significativas en la distribución según los tipos de personalidad, con un TE pequeño ( $V$ de Cramer=.19); las mujeres con el tipo S obtuvieron una frecuencia más elevada de esta categoría (51.5\%) que las de otros tipos de personalidad, principalmente con las del tipo I (25\%).

En la categoría Orden social, las mujeres presentaron diferencias significativas en la distribución según los tipos de personalidad, con un TE pequeño ( $V$ de Cramer=.2); las mujeres con el tipo I obtuvieron una frecuencia más elevada de esta categoría de constructos (22\%) que las de otros tipos de personalidad, concretamente con las de los tipos $C(0 \%)$ y $E(4 \%)$.

Por otro lado, en el área Intelectual se encontraron diferencias significativas en la distribución de frecuencias según los tipos de personalidad tanto en la muestra de mujeres, con un TE pequeño ( $V$ de Cramer=.17), como en la de los hombres, con un TE pequeño ( $V$ de Cramer=.24). Los estudiantes de ambos sexos con el tipo de personalidad I obtuvieron una frecuencia más alta de estos constructos (54\% en los hombres y $45 \%$ en las mujeres) en comparación con los de otros tipos de personalidad.

En el área Personal, los hombres presentaron diferencias significativas en la distribución de frecuencias según los tipos de personalidad, con un TE pequeño ( $V$ de Cramer=.18). Los hombres con el tipo de personalidad $C$ obtuvieron un porcentaje más alto (27\%) de estos constructos (Autoafirmación y/o realización, y Responsabilidad) que los de otros tipos de personalidad.

En cuanto al área Social, se encontraron diferencias significativas en la distribución de frecuencias según los tipos de personalidad en la muestra de hombres, con un TE pequeño ( $V$ de Cramer=.17), y en la de mujeres, con un TE pequeño ( $V$ de Cramer=.16). Los hombres con el tipo $C$ presentaron una mayor frecuencia de constructos en esta área (73\%) en comparación con los de otros tipos de personalidad, mientras que en las mujeres fue el tipo $\mathrm{S}$ el que presentó un porcentaje más elevado $(60 \%)$ en relación con otros tipos de personalidad.

\section{Conclusiones}

El análisis de la asociación entre las temáticas de los constructos vocacionales y los tipos de personalidad de Holland del alumnado mostró asociaciones significativas en la categoría Desarrollo intelectual en ambos sexos, las categorías Creatividad, Contribución social y Orden social solo en la muestra de mujeres y los constructos del área Personal únicamente en los hombres.

Los estudiantes de ambos sexos con el tipo de personalidad I presentaron una frecuencia más alta de constructos en la categoría Desarrollo intelectual (42\%) en comparación con los de otros tipos de personalidad, especialmente con A (14\%), C (14\%) y S (19\%). El alumnado con el tipo de personalidad I —el cual está relacionado con la intelectualidad, racionalidad y curiosidad, entre otras características - elaboró con más frecuencia constructos de la categoría Desarrollo intelectual, que se refiere a la realización de actividades mentales, cognitivas, de aprendizaje, la necesidad de estudio o la cualidad de ser inteligente. Aunque los resultados de las asociaciones entre la categoría Desarrollo Intelectual y los tipos de personalidad mostraron diferencias significativas en ambos sexos, se observaron algunas diferencias, tales como que los hombres con el tipo I presentaron un porcentaje más alto (45\%) que las mujeres con este mismo tipo de personalidad (37\%) y que en los 
hombres la frecuencia más baja de esta categoría la obtuvo el tipo $C(0 \%)$, en cambio, en las mujeres fue el tipo A (13\%).

Respecto a la categoría Creatividad, los resultados mostraron patrones de asociación con los tipos de personalidad diferentes según el sexo. Las mujeres con el tipo A elaboraron con más frecuencia constructos de esta categoría (22\%) que aquellas clasificadas en otros tipos de personalidad, concretamente en $S(5 \%)$ e I (8\%), en cambio, en la muestra de los hombres no se encontraron diferencias significativas en la distribución de esta categoría de constructos según los tipos de personalidad. Las mujeres de la muestra con el tipo de personalidad A -el cual está asociado, entre otras características, con la imaginación, intuición, sensibilidad y creatividadelaboraron con más frecuencia constructos en los que se describen actividades dirigidas a desarrollar ideas, conceptos, objetos, la imaginación y la creatividad.

Los resultados de la asociación entre los tipos de personalidad y las categorías Contribución social y Orden social mostraron diferencias de género. En la muestra de las mujeres se encontraron asociaciones significativas, mientras que en la de los hombres no. Las mujeres con el tipo de personalidad $S$ presentaron con más frecuencia constructos de Contribución social (51.5\%) en comparación con las de otros tipos de personalidad, principalmente con I (25\%). Pero también fue la categoría más frecuente en las mujeres con los tipos A (40\%), E (38\%) y C (35\%), aunque con una frecuencia más baja que en las del tipo $\mathrm{S}$. Las mujeres de la muestra con el tipo de personalidad $\mathrm{S}-$ caracterizado por la sociabilidad, extraversión y el servicio a los demás- elaboraron con más frecuencia constructos de Contribución social, los cuales se refieren a la motivación por ofrecer un servicio profesional para ayudar en el desarrollo y bienestar de otras personas y de la sociedad. Con respecto a la categoría Orden social, las mujeres con el tipo de personalidad I presentaron con más frecuencia este tipo de constructos (22\%) en comparación con las de otros tipos. Esta categoría está formada por constructos que tratan sobre actividades dirigidas a la administración de la justicia, investigación de delitos y ejecución de sentencias.

En cuanto a las categorías del área Personal, los resultados indicaron diferencias de género. En la muestra de los hombres se obtuvo una asociación significativa entre la distribución de frecuencias en esta área y los tipos de personalidad, en cambio, en la de mujeres no. Los hombres con el tipo de personalidad $\mathrm{C}$ elaboraron con más frecuencia constructos de esta área -específicamente Autoafirmación y/o realización, y Responsabilidad (27\%)- en comparación con los de otros tipos de personalidad. Las personas $\mathrm{C}$ se asocian con ser ordenadas, metódicas, prácticas, conformistas, eficientes y con capacidades administrativas, entre otras características. Los hombres de la muestra con este tipo de personalidad elaboraron más constructos que hacen referencia a la responsabilidad, la expresión de las propias ideas y capacidades, y al logro de las expectativas personales y vocacionales.

Estos resultados confirman la conjetura que se había planteado sobre la existencia de aspectos convergentes entre las temáticas de los constructos vocacionales y los tipos de personalidad de Holland en la muestra de estudiantes. No obstante, estos hallazgos indican que estas asociaciones difieren según el sexo.

Las diferencias de género han sido una constante en la línea de investigación sobre el comportamiento vocacional (Rojewski, 2005). Con respecto a la teoría de los tipos de personalidad de Holland, existe mucha evidencia sobre este tipo de diferencias; las mujeres tienden a obtener puntuaciones más altas en S y A, y más bajas en I y R que los hombres (Spokane y Cruza-Guet, 2005). En la estandarización mexicana del SDS (Holland et al., 2005), el tipo R fue el más frecuente en los hombres y en las mujeres los tipos A y S. Los resultados de la presente investigación muestran diferencias de género semejantes, un porcentaje significativamente más elevado de mujeres se clasificaron en el tipo de personalidad $S$ que los hombres y estos obtuvieron un porcentaje 
significativamente más alto en el tipo $\mathrm{R}$ que las mujeres, de las cuales ninguna presentó este tipo de personalidad.

Por otro lado, las investigaciones sobre la RV que han analizado la temática de los constructos vocacionales han identificado también diferencias de género (Fernández et al., 2017; Rivas y Ardit, 1985, citado en Martínez, 2005). Fernández et al. (2017) encontraron en una muestra de estudiantes indígenas de México que los constructos del área Social y de la categoría Contribución social fueron significativamente más frecuentes en las mujeres que en los hombres, y que estos últimos presentaron una frecuencia significativamente más alta de constructos en el área Laboral (que hacen referencia a la retribución económica, seguridad laboral e independencia en el trabajo) que las mujeres. Por su parte, Rivas y Ardit (1985, citado en Martínez, 2005) identificaron en una muestra de estudiantes preuniversitarios españoles una frecuencia mayor en las mujeres de constructos orientados al ámbito social, como ayudar a las personas, mientras que en los hombres una frecuencia más elevada de constructos sobre aspectos extrínsecos, prácticos y con recompensas financieras. Los resultados de la presente investigación señalan diferencias de género semejantes, las mujeres presentaron un porcentaje significativamente más elevado de constructos en el área Social que los hombres y estos un porcentaje significativamente más elevado de constructos en el área Laboral que las mujeres, no obstante, los hombres obtuvieron un porcentaje más elevado de constructos en el área Intelectual que las mujeres, resultado que no se había identificado en los estudios anteriores.

Aunque no se han encontrado investigaciones anteriores sobre la asociación entre los tipos de personalidad de Holland y las temáticas de los constructos vocacionales, con las cuales contrastar los resultados obtenidos; se puede observar cierto paralelismo con los resultados de los estudios que han analizado la asociación entre los tipos de personalidad y los valores (Holland et al., 2005; Rottinghaus y Zytowski, 2006). Las primeras investigaciones sobre este tema mostraron que cada uno de los seis tipos de personalidad se asociaban con determinados valores, aunque en ocasiones algunos valores lo hacían con más de un tipo de personalidad (Holland et al., 2005; Nauta, 2010). La investigación más reciente de Rottinghaus y Zytowski (2006) mostró algunos aspectos convergentes entre los intereses RIASEC y los valores laborales, que diferían según el sexo. De manera similar, la presente investigación ha identificado algunas asociaciones entre las temáticas de los constructos vocacionales y los tipos de personalidad, y que estas asociaciones presentan diferencias de género. Asimismo, se ha encontrado que algunos constructos vocacionales se asocian con más de un tipo de personalidad; concretamente la categoría Contribución social, que fue la más frecuente en las mujeres con el tipo $S$, pero también presentó porcentajes relativamente altos en las mujeres $A$ y $E$. En el modelo hexagonal estos tipos son adyacentes del $\mathrm{S}$ y, por consiguiente, están más relacionados.

Los resultados de esta investigación revelan que la RV y el SDS proporcionan información distinta sobre los intereses, preferencias y valores vocacionales de los estudiantes, y que existen algunos aspectos de esta información que se asocian. La RV ofrece información desde un enfoque cualitativo e idiográfico y el SDS desde el cuantitativo y nomotético, que se pueden complementar para alcanzar una comprensión más rica, profunda y personal sobre las preferencias y valores vocacionales del alumnado. Aparte de esta complementariedad de la información personal que ofrecen ambos instrumentos, desde el modelo constructivista se ha sugerido que la tipología de Holland proporciona un patrón integrador de intereses vocacionales, que puede ser útil para que el alumnado organice su sistema de constructos (Savickas, 1997).

Las asociaciones que se han identificado entre la temática de los constructos vocacionales y los tipos de personalidad contribuyen al conocimiento sobre la definición de los tipos de personalidad de Holland, específicamente de los tipos I, A y S, en esta población de estudiantes y aportan evidencia de validez concurrente del sistema de clasificación de los constructos vocacionales utilizado. Por otro lado, las diferencias de género reflejan la influencia de los factores socioculturales en los intereses, motivaciones y valores personales con respecto al ámbito laboral. En la práctica de la orientación vocacional, la información sobre las diferencias de género y las asociaciones entre la temática de los 
constructos vocacionales y los tipos de personalidad es útil para la integración e interpretación de los datos obtenidos en la RV y el SDS en el proceso de exploración vocacional del alumnado.

Entre las limitaciones de la investigación, se encuentra el pequeño tamaño de la muestra de estudiantes, especialmente de los que se clasificaron en los tipos de personalidad $\mathrm{C}$ y $\mathrm{R}$, lo que cuestiona las conclusiones que se pueden establecer sobre la asociación entre estos tipos y la temática de los constructos vocacionales. Se requieren más investigaciones con muestras más amplias para comprobar las asociaciones identificadas y, en concreto, entre el tipo de personalidad C y los constructos del área Personal (Autoafirmación y/o realización, y Responsabilidad) en los hombres. Otra limitación de la investigación consiste en que en el análisis no se tuvo en cuenta el alumnado con puntuaciones iguales en más de un tipo de personalidad, lo cual genera un sesgo en la muestra; sería interesante abordar, en próximas investigaciones, el análisis de la asociación entre la temática de los constructos vocacionales y los tipos de personalidad en este alumnado, el cual se caracteriza por presentar niveles más bajos de diferenciación en el tipo de personalidad (Holland et al., 2005). Otra cuestión que no se ha llevado a cabo en esta investigación hace referencia al análisis de la asociación entre los constructos vocacionales y los tipos de personalidad de Holland, el cual se realizó únicamente desde una metodología cualitativa; la técnica de la rejilla permite, además, análisis cuantitativos a partir de las valoraciones que las personas hacen de los constructos y elementos, el estudio de la relación entre estas valoraciones y las puntuaciones obtenidas en el SDS puede aportar información complementaria sobre este tema.

\section{Referencias bibliográficas}

Alonso-Tapia, J. (2012). Evaluación psicopedagógica y orientación educativa (Vol. II). Integración social, evaluación del entorno instruccional y valoración de programa. Madrid: Síntesis.

American Psychological Association (APA). (2017). Ethical Principles of Psychologists and Code of Conduct. Recuperado de https://www.apa.org/ethics/code/ethics-code-2017.pdf

Bodden, J. L. (1970). Cognitive complexity as a factor in appropriate vocational choice. Journal of Counseling Psychology, 17, 364-368. https://doi.org/10.1037/h0029677

Botella, L., y Feixas, G. (2008). Teoría de los constructos personales: aplicaciones a la práctica psicológica (edición electrónica). Barcelona: FPCEE Blanquerna. doi: 10.13140/RG.2.1.1046.2482

Feixas, G., y Cornejo, J. M. (1996). Manual de la técnica de la rejilla mediante el programa RECORD v. 2.0. Barcelona: Paidós.

Fernández, M. T., Tuset, A. M., y Cuervo, M. (2017). La técnica de la rejilla en la evaluación de las aspiraciones y constructos vocacionales. Psicología Educativa, 23, 53-62. doi: http://dx.doi.org/10.1016/j.pse.2017.02.002

Hay, R. G. (1985). How individuals construe occupations: A validation study of a career grid (Tesis doctoral). lowa State University. Recuperado de https://lib.dr.iastate.edu/rtd/12071/

Holland, J. L. (1997). Making vocational choices: A theory of vocational personalities and work environments ( $3^{\mathrm{a}} \mathrm{ed}$ ). Odessa, FL: Psychological Assessment Resources.

REOP. Vol. 31, nº1, 1er Cuatrimestre, 2020, pp. 10 - 25 [ISSN electrónico: 1989-7448] 
Holland, J. L., Fritzsche, B. A., y Powell, A. B. (2005). SDS Búsqueda Autodirigida. Manual Técnico. Ciudad de México: El Manual Moderno.

Izcara, S. P. (2007). Introducción al muestreo. Cd. de México: Porrúa.

Kelly, G. (1955/1991). The psychology of personal constructs (2 vols.). Nueva York: Norton (reimpreso por Routledge, London, 1991).

Martínez, B. C. (2005). Estructuración cognitiva del mundo vocacional (Tesis doctoral). Universitat de Valencia. Recuperado de www.tdx.cat/bitstream/10803/10225/1/martinez.pdf

Martínez, J. M. (2007). El asesoramiento vocacional y profesional a través del Self-Directed Search (SDS). Revista Electrónica de Investigación Psicoeducativa, 5(11), 233-258.

Mau, W. (1997). Assessing the cognitive complexity of vocational value constructs using the career grid: A comparison of the expressed and supplied methods. Measurement $y$ Evaluation in Counseling y Development, 29(4), 202-214.

Nauta, M. M. (2010). The development, evolution, and status of Holland's theory of vocational personalities: Reflections and future directions for counseling psychology. Journal of Counseling Psychology, 57(1), 11-22. doi: 10.1037/a0018213

Neimeyer, G. J. (1989a). Personal construct systems in vocational development and informationprocessing. Journal of Career Development, 16(2), 83-96.

Neimeyer, G. J. (1989b). Applications of repertory grid technique to vocational assessment. Journal of Counseling and Development, 67(10), 585-589.

Neimeyer, G. J. (1992a). Personal constructs in career counseling and development. Journal of Career Development, 18(3), 163-173.

Neimeyer, G. J. (1992b). Personal constructs and vocational structure: A critique of poor reason. En R. A. Neimeyer y G. J. Neimeyer (Eds.), Advances in Personal Construct Psychology (Vol. 2, pp. 91-120). Greenwich, CT: JAI Press.

Padilla, M. T. (2001). La rejilla de constructos personales: un instrumento para el diagnóstico y la orientación. Ágora Digital, 2, 1-12.

Paszkowska-Rogacz, A., y Kabzińska, Z. (2012). Applications of Kelly's personal construct theory to vocational guidance. Psychology Research, 2(7), 408-421.

Rivas, F. (1998). Psicología vocacional: Enfoques del asesoramiento. Madrid: Morata.

Rivas, F., Rocabert, E., y López, M. L. (1998). Manual del SAV-R y SAVI-2000. Sistemas de autoayuda y asesoramiento vocacional. Valencia: Servicios de Asesoramiento Vocacional y Educativo.

Rojewski, J. W. (2005). Occupational aspirations: Constructs, meanings, and application. En S. D. Brown y R. W. Lent (Eds.), Career Development and Counseling: Putting Theory and Research to Work (pp. 131-154). Hoboken, NJ: John Wiley y Sons.

Rottinghaus, P. J., y Zytowski, D. G. (2006). Commonalities between adolescents' work values and interests. Measurement and Evaluation in Counseling and Development, 38(4), 211-221. doi: 10.1080/07481756.2006.11909783 
Sánchez, P. A., y Valdés, A. A. (2007). Teoría y práctica de la orientación en la escuela. Un enfoque psicológico. Ciudad de México: El Manual Moderno.

Saul, L. A., López-González, M. A., Moreno-Pulido, A., Corbella, S., Compañ, V., y Feixas, G. (2012). Bibliometric review of the repertory grid technique: 1998-2007. Journal of Constructivist Psychology, 25, 112-131.

Savickas, M. L. (1997). Constructivist career counselling: Models and methods. En R. Neimeyer y G. Neimeyer (Eds.), Advances in Personal Construct Psychology (Vol. 4, pp. 149-182). Greenwich, CT: JAI Press.

Spokane, A. R., y Cruza-Guet, M. C. (2005). Holland's theory of vocational personalities in work environments. En S. D. Brown y R. W. Lent (Eds.), Career Development and Counseling: Putting Theory and Research to Work (pp. 24-41). Hoboken, NJ: John Wiley y Sons.

Taylor, J. M. (2016). Using personal construct psychology: Constructing a career with an Asian American client. En L. A. Busacca y M. C. Rehfuss (Eds.) Postmodern Career Counseling: $A$ Handbook of Culture, Context, and Cases (pp. 189-200). Alexandria, VA: American Counseling Association.

Vilhjálmsdóttir, G. (2010). Occupational thinking and its relation to school dropout. Journal of Career Development, 37(4), 677-691. doi: 10.1177/0894845309357052

Winer, J. L., Warren, G. D., Dailey, K. C., y Hiesberger, J. (1980). Complexity of judgment of occupational titles among Holland types. The Vocational Guidance Quarterly, 29, 12-24. doi: https://doi.org/10.1002/j.2164-585X.1980.tb01016.x

Fecha de entrada: 28 noviembre 2018

Fecha de revisión: 16 diciembre 2019

Fecha de aceptación: 03 febrero 2020 